\title{
Instruments of change for academic tool development
}

\author{
Scientific progress has always been driven by the ability to build an instrument to answer a specific question. But \\ spreading the news of how to replicate that tool is an evolving art, ripe for an open-source revolution.
}

\section{Georg E. Fantner and Andrew C. Oates}

$\mathrm{n}$ the early days of scientific endeavour, the simplicity of instruments was such that a scientist's peers could readily adopt his or her designs for their own experiments. However, with the increasing complexity of specialized scientific instruments, conveying the research behind a given device is no longer straightforward. Progress in science is now paired with a certain fragmentation: the people studying a particular question are rarely skilled enough to build the instrument required to solve it. And yet we feel it is essential that scientists continue to innovate in scientific instrumentation. The challenge we face as a community is how best to disseminate our innovations. Taking inspiration from the open-source software movement, we have developed one way of meeting this challenge head on - and much like our tools, we're eager to share it with the world.

\section{A problem to be solved}

In an academic setting, it is becoming increasingly difficult to justify the costs and time required to develop a prototype instrument that is used in only one lab. To leverage these costs, many universities have focused on disseminating the technologies developed in their research labs through patenting and subsequent commercialization via start-ups or licensing (Fig. 1, red route).

This process begins with the fabrication of a prototype instrument that proves the design's potential. Often such prototypes are temperamental and require continued maintenance by the inventor. Once a patent has been filed, a company interested in making the device commercially viable will therefore need to undertake further research to broaden its appeal and utility. If successful, this model can work to the advantage of universities, commercial entities and the scientific community. Unfortunately, often this model is ineffective for several reasons, including long delays in the commercialization process and high patent costs that are often not recuperated.

Many innovations that clearly benefit science are not suitable for the traditional

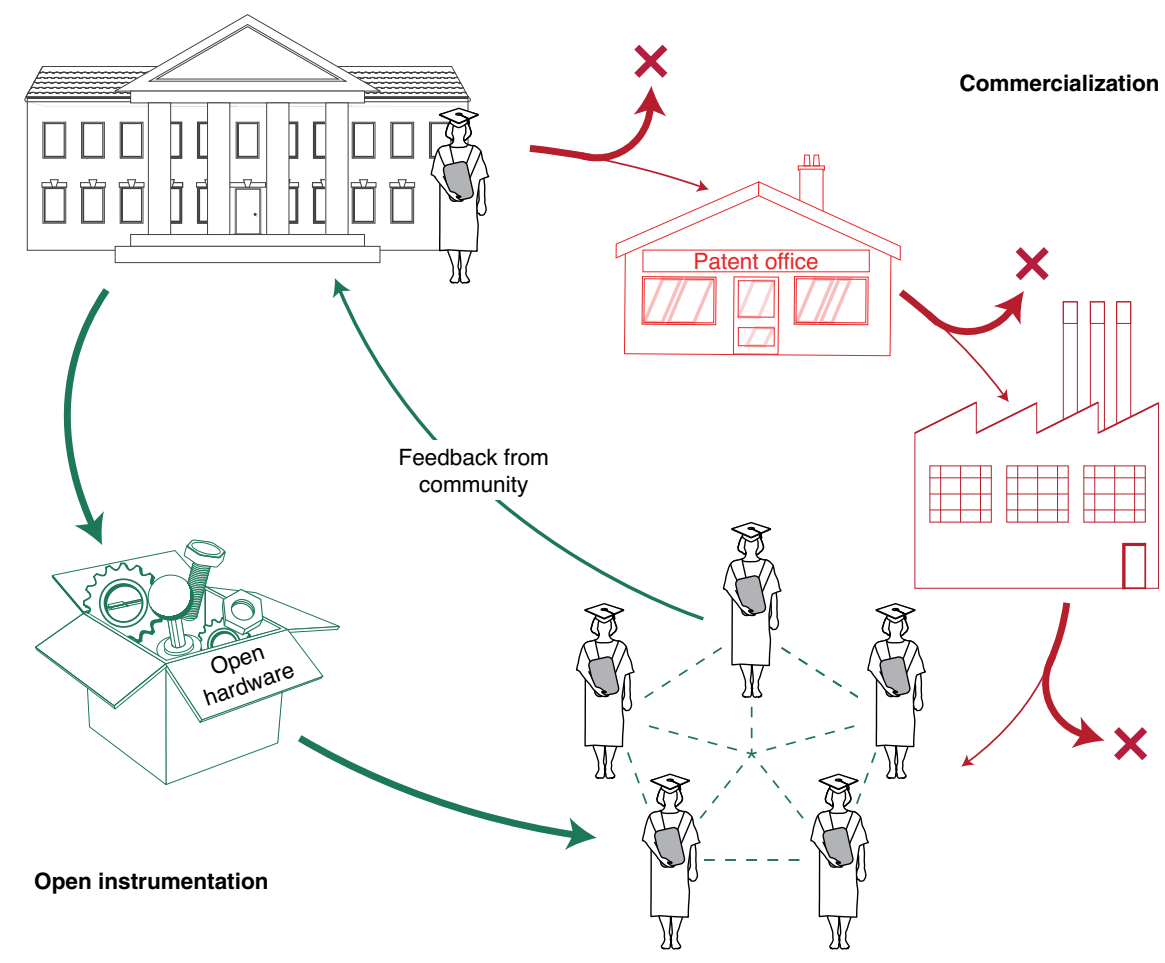

Fig. 1 | Two routes to disseminate innovation in academic instrumentation to the broader scientific community. Traditional commercialization (top right, red) involves filing patents and partnering with industry to distribute new inventions. This route can be productive in terms of reaching a large audience, but it comprises several steps, each of which has a substantial chance of failing. Direct dissemination through open hardware (bottom left, green) offers an alternative path. It has the advantages of avoiding intermediaries, and fostering relationships between inventors and users. This in turn facilitates feedback from users to inventors, which can speed up the innovation cycle.

commercialization pathway. This could be because an instrument is highly specialized, claiming only a small market share. Moreover, no matter how useful the instrument, if it is not easily protected by patents, commercial partners are hesitant to take on the investment for product development and market introduction. Sometimes manufacturers even purposefully delay innovations to maintain the profitability of existing products. For these and other reasons, we argue that many highly useful innovations never reach the scientific community. This is a tremendous loss of opportunity and waste of public resources.

\section{Open-source inspiration}

Recently an alternative approach has emerged, which we term open-hardware instrumentation. The idea is that the lab in which the invention is devised also takes care of the first steps of its dissemination (Fig. 1, green route). Early adopters of this idea include the open light-sheet microscope project (OpenSPIM; https://openspim. org/) by Pavel Tomančak ${ }^{1}$, our initiative in 
open high-speed atomic force microscopy (OpenAFM; https://www.epfl.ch/labs/lbni/ openhardware/) and the recent open-source Förster resonance energy transfer platform (smfBox) run by Timothy Craggs ${ }^{2}$.

This model is similar in spirit to open-source software, a success story for how relevant innovations can be shared quickly with the broader scientific community. Open-source software has significantly advanced progress and innovation speed in areas such as image processing ${ }^{3}$, machine learning ${ }^{4}$ and big data management, primarily by harnessing and coordinating the efforts of many individuals into teams working collectively towards ambitious goals. It grants developers access to large codebases, which offer solutions to specific problems, and in turn speed up innovation.

The success of open-source software has relied heavily on the development of tools for collaboration, such as GitHub (https:// github.com), standards for documentation, a community-based support system and licensing models. Indeed, open-source software has led to new commercial activities, either with companies building their software suites on open-source code, or businesses providing service and support for open-source applications ${ }^{5}$.

\section{Benefits and challenges}

There are multiple advantages for the scientific community, as well as funding agencies, to adopt a similar model for open instrumentation. Many hardware developments that are not suitable for the traditional commercialization pathway could become available to the research community. Open hardware not only provides access to existing developments but access to well-designed and well-documented tools also serves as a starting point for further innovation. This means that an academic's time can be spent on innovation, rather than recreating other scientists' technologies, resulting in shorter innovation cycles and more efficient use of research funding. This is particularly important for research areas in which progress moves faster than the traditional commercialization cycle. And sharing one's work through open instrumentation can lead to quicker uptake of an individual's technologies, which may bring with it acclaim and citations.

Open hardware also presents an opportunity for education. By making their prototype instruments suitable for sharing, students acquire the skills essential to commercialize their innovations or pursue careers in industry. Students sharing their instrument designs with others receive rapid feedback - and perhaps even hands-on help - advancing their projects faster.
Making an open-hardware instrument successful, however, involves overcoming several challenges, some of which are shared by the open-source software movement. The need for clear documentation, project continuity and licensing models is the same, and some of the solutions developed for software can be readily adopted.

But hardware creates a distinct set of challenges because instruments need to be physically built rather than digitally copied. Sourcing parts can be difficult, as complex instruments often require specialized components of high quality. Solutions could include providing ready-to-fabricate drawings in a format suitable for rapid prototyping services, or by making kits available at cost. Assembling parts also might require specialized knowledge to ensure that the final instrument performs optimally. Hence, detailed assembly and calibration instructions are required. The broad and long-term availability of third-party components also needs to be accounted for in the design process.

Life-cycle management for open hardware is also significantly different from open-source software. In contrast to software, hardware can show signs of use, and performance degrades over time, requiring maintenance and repair. Finally, because hardware cannot be updated as easily as software, the rate of revisions should be limited to avoid large inhomogeneities in the user base within the community.

Similar issues are faced by the maker movement, which has enabled a new trend in citizen science by making simple scientific tools available for everyday people at low $\operatorname{cost}^{6}$. Broadly available resources such as three-dimensional printers and cheap reconfigurable hardware platforms such as Arduino (https://www.arduino. $\mathrm{cc} /$ ) and Raspberry Pi (https://www. raspberrypi.org/) can be used in people's garages or hackerspaces to provide a basic level of scientific equipment. However, the relatively small investment in parts and time for these simpler instruments makes the aforementioned challenges less severe.

One area in which open hardware has found increasing success is open electronics. A high degree of design standardization, the wide availability of affordable components and the existence of many third-party manufacturers has made the broader adoption of open hardware in electronics manageable. Open electronics has successfully made use of best practices from open-source software to store, maintain and disseminate the projects. One successful example is the CERN open-hardware repository (https://ohwr.org/).

\section{Early success stories}

Measurement instruments developed in an academic environment are often sophisticated, requiring knowledge of electrical, mechanical, optical, software and sample-mounting techniques. For this reason, sharing such developments via open hardware is still uncommon, yet some pioneering examples exist.

The OpenSPIM (https://openspim. org/) spearheaded by Pavel Tomančak solved the sourcing challenge by using almost exclusively commercial parts, with the few custom parts required being easy to fabricate. Video tutorials rigorously document the steps required for assembly, and maintenance and support are organized by a community of more than a dozen labs connected via a forum (image.sc). The no-frills design and choice of commercial parts makes the barrier for adoption and subsequent modifications low.

An example of a different strategy for enabling researchers to adopt an open-hardware project is our own OpenAFM project (https://www.epfl.ch/labs/lbni/ openhardware/). This initiative was initially focused on optimizing performance and not specifically designed for easy sharing. Consequently, the instrument contains a large number of custom components to achieve top performance, which makes sourcing and assembly more difficult.

Making a copy of the OpenAFM device in one's own lab is therefore not trivial, and the barrier for subsequent modifications is high. To overcome this barrier, we came up with a different strategy: we actively support labs interested in building our device by holding open-hardware workshops every year. Participants in these workshops spend one week in our lab, where they receive a kit containing all the parts required for assembly, the costs of which constitute the workshop participation fee. The parts are sourced collectively by the workshop organizers, which helps keep the fees low. Over the course of the week, the participants learn how to assemble, debug and test the instrument, ensuring that at the end of the workshop each participant leaves with a working device, full documentation, and the skills to use, service and modify their new toy ${ }^{7}$.

After several years of organizing these workshops, we see the benefits, but also the challenges in sharing one's instrumentation as open hardware. On the positive side, it has led to collaborations, joint publications ${ }^{8}$ and citations. Participants have told us that they acquired unique insights into the instruments, which accelerated their research back home. Doctoral students who have organized the workshop found it to be a rewarding and educational experience. 
On the downside, organizing the workshops is a substantial time investment, and maintaining a consistent quality as students graduate requires timely training of the next generation. Although the time spent organizing these workshops can be counted as dissemination (a required activity for most public grants), it is not always sufficiently appreciated by thesis committees or those responsible for career advancement. In the current climate where publishing remains the primary measure of success, it can be difficult to motivate young scientists to invest their time in open science.

\section{Lessons learned}

The scientific open-hardware movement is still in its infancy. Although several 'low tech' open instruments have been very successful in democratizing science ${ }^{9}$ and engaging the public in community science ${ }^{10,11}$ (see also https://www.citizensciencealliance.org), few research-grade open-hardware instruments exist. And some suffer the stigma of being 'cheap'.

To tap into the real potential of scientific open hardware, researchers should share the designs for their state-of-the-art instruments in an open-science fashion. As more high-end research results are obtained with open-hardware instruments, scientists, as well as university leadership, will come to appreciate this potential. We should therefore strive to make it easier for researchers to make their instrument designs openly accessible.

Many universities currently provide financial and staffing support for researchers to store their research data and publish their findings in open-access journals. However, rarely is support for open-hardware projects available. University technology-transfer offices could play a vital role here by providing support in choosing the right licensing model, helping to avoid liabilities and ensuring no third-party rights are violated.

Maintaining an open-hardware project will always require a significant amount of effort. When the open-hardware project is successful, the effort required can exceed what we might reasonably expect academics to invest. Here, an opportunity exists for innovative service enterprises. Entrepreneurs could take over the management of the open-science project, providing consulting, training, workshops or even built-to-order and service contracts for the open-hardware instruments. Similar business models have been successful in open-source software, and some start-ups are appearing in the realm of open hardware (http://www.easy-phi.ch/index.php).

The open-hardware movement undoubtedly offers commercial opportunities $^{12}$, but it has also come under fire from those who feel that it challenges the current industry model. Although there are certainly some areas where open-hardware projects could be in direct competition with commercial solutions, we argue that the benefits of the open-hardware movement to the instrumentation industry outweigh the costs of competition.

First, open hardware generally targets a different audience than commercial instruments. Open hardware typically attracts people interested in building and modifying their instruments. As such, many open-hardware projects do not build everything from scratch, but either add onto existing commercial instruments or use a collection of commercial components to build a tool with new functionality. Hence, these open-hardware projects increase the demand for commercial components. Companies could market the inclusion of generic open-hardware interfaces on their commercial instruments, similar to scripting languages already provided by many suppliers.

Second, companies could themselves make use of open-hardware projects that can serve as testbeds or incubators before they decide to invest the resources necessary to develop a new technology into a commercial product. Indeed, one could imagine commercial companies supporting academic open-hardware projects for cost-effective pre-development and market testing. Eventually, companies might choose to develop their own products making use of open-hardware developments, as has been the case with open-source software.

Scientists wanting to make their instruments available as open hardware should avoid some pitfalls that may limit their chances of success. One mistake is to try to mimic a commercially available instrument. If the same functionality exists commercially, there is no need to re-develop it. The development cost almost always outweighs the purchase price so not many users will adopt the open-hardware project. A related pitfall is trying to make an open-hardware instrument purely for the sake of lowering the cost. Often open-hardware instruments seem cheaper because many costs faced by commercial manufacturers are hidden, including development, support, sales and marketing costs. Many of these costs, however, still exist in an academic setting - in the salaries of PhD students, for example.

Another mistake is trying to make an instrument that serves everybody and thereby competes with commercial multipurpose instruments. The strength of open-hardware lies in specificity, creativity, flexibility and innovation speed. Trying to make an all-purpose tool requires lots of development effort with little intellectual contribution. It often requires making inevitable trade-offs that limit the performance that made the initial instrument unique. Besides, an instrument that offers too many possibilities becomes increasingly complex, which again makes it difficult for others to build and maintain.

The most significant risk of all, however, is disseminating the open-hardware project too early. Even though an instrument might work well in the hands of the people who have built it, it doesn't mean it is usable by others. It is essential to develop the open-hardware instrument to a level at which it is robust enough to share. Also, compared with open-source software, which can be replaced with a single download, the upgrade process for open hardware is much more difficult because it involves building a new system. Releasing an open-hardware project too early can mean that people will be hesitant to adopt the project or, if they do so, they might be disappointed in the time and money invested. This can permanently damage the reputation of the open-hardware project. This pitfall can be avoided by starting small and close to home. By first sharing one's instrument with a nearby lab, one can identify and resolve issues quickly before sharing the instrument globally.

\section{The way forward}

As measurements and the instruments that facilitate them are at the heart of many discoveries, the trend towards making science more open, accessible and reproducible should also include instrument development. The advantages for the lab, the scientific community and the funding agencies are compelling. There is also a looming regulatory requirement that can be satisfied by open instrumentation. As funding agencies move towards mandating open-access publishing, the idea of open hardware becomes more attractive. Indeed, if the outcome of research is a scientific instrument with unique capabilities, there may soon be an obligation to disseminate it openly, just as we do with more traditional results.

Over the past decade, several open-hardware projects have appeared with varying levels of success. Some have turned into long-standing projects with a loyal following, whereas others have been abandoned. Happily, there is help at hand for new developers from some of the early success stories of open hardware. Projects like the CERN open hardware repository (https:// ohwr.org/) or the Open Source Hardware Association (https://www.oshwa.org) 
provide useful resources for academic instrument developers who want to share the results of their research.

If open instrumentation is to find broad adoption in the scientific community, however, it will require a concerted effort. On the academic level, contributions to open-hardware projects need to be valued by thesis and promotion committees. Publishers should require detailed construction documentation for papers that rely on custom hardware, and funding agencies should mandate open-hardware dissemination as they have open-access publication and open data repositories. And although such a transition will undoubtedly take time and effort, unleashing the largely untapped potential of academic open hardware is in the best interest of all members of the broader research community.

Georg E. Fantner (D) $1 \times$ and Andrew C. Oates ${ }^{2}$ ${ }^{1}$ Laboratory for Bio- and Nano-Instrumentation, Interfaculty Institute for Bioengineering, École Polytechnique Fédéral de Lausanne, Lausanne, Switzerland. ${ }^{2}$ Timing, Oscillations, Pattern

Laboratory, Interfaculty Institute for Bioengineering, École Polytechnique Fédéral de Lausanne, Lausanne, Switzerland.

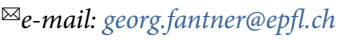

Published online: 9 April 2021

https://doi.org/10.1038/s41567-021-01221-3
References

1. Pitrone, P. G. et al. Nat. Methods 10, 598-599 (2013).

2. Ambrose, B. et al. Nat. Commun. 11, 5641 (2020).

3. Schindelin, J. et al. Nat. Methods 9, 676-682 (2012).

4. Zaharia, M. et al. Commun. ACM 59, 56-65 (2016).

5. Faletski, I. Yes, you can make money with open source. Harvard Business Review https://hbr.org/2013/01/ yes-you-can-make-money-with-op (2013).

6. Diederich, B. et al. Nat. Commun. 11, 5979 (2020).

7. Biophysical Society TV: Open Hardware, Academic Instrument Development in the Age of Open Science (Websedge, 2019).

8. Parsons, E. S. et al. Nat. Commun. 10, 2066 (2019).

9. Harnett, C. IEEE Instrum. Meas. Mag. 14, 34-38 (2011).

0. Gura, T. Nature 496, 259-261 (2013)

11. Ephraim, R. K. D. et al. Am. J. Trop. Med. Hyg. 92, 1253-1256 (2015).

12. Li, Z. \& Seering, W. Proc. Des. Soc. Int. Conf. Eng. Des. 1, 2239-2248 (2019)

Competing interests

The authors declare no competing interests. 